\title{
Vaccine Hesitancy, Refusal and Access Barriers: The Need for clarity in terminology
}

Helen Bedford ${ }^{\text {a, }}$ Katie Attwell ${ }^{b, c,}$, Margie Danchin ${ }^{d}$, Helen Marshall e, Paul Corben ${ }^{f}$, Julie Leask ${ }^{g}$,

a Faculty of Population Health Sciences, UCL Great Ormond Street Institute of Child Health, 30 Guilford Street, London WC1N 1EH, United Kingdom

b Sir Walter Murdoch School of Public Policy and International Affairs, Murdoch University, Murdoch WA 6150, Australia

c Wesfarmers Centre of Vaccines and Infectious Diseases, Telethon Kids Institute, Western Australia

d Murdoch Children's Research Institute, Flemington Road, Parkville, Victoria, 3052 Australia

e Robinson Research Institute and Adelaide Medical School, University of Adelaide and Women's and Children's Health Network, Adelaide, South Australia 5006, Australia

${ }^{f}$ North Coast Public Health Unit, Port Macquarie Community Health Campus, PO Box 126 Port Macquarie NSW 2444 Australia

gSchool of Public Health, University of Sydney, Edward Ford Building A27, Camperdown NSW 2006 Australia

In most countries, although uptake of childhood vaccines is generally high, pockets of undervaccination pose a continued threat to individual protection and population immunity, with some of these pockets the foci of recent disease outbreaks [1,2]. Vaccination safety scares have led to long-term reductions in coverage, as evidenced by reduced coverage of MMR vaccine post the suggested association with autism in many countries [3, 4]. Increasingly, experts and commentators have used the term 'vaccine hesitancy' to explain sub-optimal vaccination coverage, regardless of the contextual contributory causes [5]. We contend that using the term 'vaccine hesitancy' to explain all partial or non-vaccination is inaccurate, and risks generating solutions that are a poor match for the problem in a particular community or population. Here, we propose more precision in the use of the term 'vaccine hesitancy,' and its delineation from factors related to access and pragmatics. Only with a clear distinction between these determinants can we develop appropriate policies and strategies targeted to the specific drivers of lower-than-desired vaccination coverage in each population. 
Hesitancy is defined in the Oxford English Dictionary as "The quality or condition of hesitating; indecision, vacillation; an instance of this." To hesitate is to "hold back in doubt or indecision; to show, or speak with, indecision; to find difficulty in deciding; to scruple." These definitions portray hesitancy as a psychological state which may delay action or result in inaction. The terms "hesitant, hesitate or hesitancy" were first applied to vaccination in 1994 to describe physicians' reluctance to prescribe a vaccine. From 2004, journal articles began to relate hesitancy to parents or adult vaccination recipients, reaching a peak in 2015 with 50 articles indexed in Medline using the term.

The term "hesitancy" is now widely used in public commentary about vaccination coverage, with three problematic patterns emerging: (1) 'Vaccine hesitancy' is represented as a behaviour, even though it is a psychological state; (2) the label 'hesitancy' is applied to nonvaccination, when in fact some non-vaccinators are forthright in their refusal, and may never have been hesitant and (3) 'hesitancy' is used as the explanation for under-vaccination in a population when, in fact, the causes are related to pragmatics, access, or the failure of services or policies.

The WHO SAGE vaccine hesitancy working group (which was constituted to address the emerging phenomenon in November 2011) grappled with defining vaccine hesitancy. It rested with a behaviourally-related definition: "a delay in acceptance or refusal of vaccines despite availability of vaccination services". The group rightly noted that hesitancy is a complex and context specific phenomenon, varying across time, place and vaccines. However, its report went on to conclude that vaccine hesitancy is influenced by factors such as confidence (do not trust vaccine or provider), complacency (do not perceive a need for a vaccine, do not value vaccination), and convenience (access to vaccines)" [6]. Additionally, they considered that while some parents might still value and have access to vaccines, vaccination may be a low priority due to domestic pressures or busy lives.

We believe that adding the concept of 'convenience' (and its related notion of parental prioritisation) to a definition of hesitancy is problematic. It bundles together concepts relating to individuals' decisions (selecting out or refusing vaccines) with individual and system-level factors that may contribute to difficulties accessing vaccines. Understandably, the WHO report sought to keep service-related factors on the global agenda, ensuring that countries and services continued to be accountable for their role in ensuring high vaccination uptake. However, the settled definition perpetuates terminological imprecision. This imprecision could lead to the development of one-solution policies and interventions designed to overcome 'vaccine hesitancy' that fail to account for the range of causes of sub-optimal vaccination.

Notably, the problematic usage of the term 'vaccine hesitancy' in the media and medical literature does not give sufficient emphasis to the social determinants of vaccination. These can impede access to existing resources, and are arguably the largest contributor to under- 
vaccination. For example, a community might have low vaccination rates, assumed to be due to parents' actively refusing vaccination. In response, an agency might implement a campaign to address 'hesitancy,' only to find that the main barriers were actually inflexible and inadequate primary care services, work-place policies minimising time-off for parents, or missed opportunities.

Indeed, many studies find that access barriers are a significant reason for children not being up to date with their vaccinations $[7,8,9]$. These children are more likely to be economically disadvantaged, belong to larger families, and face practical or logistical challenges to access $[8,9,10]$. Access barriers apply at both an individual and a "system" level and are ripe for solutions to minimise them (such as lack of transport or money) by providing accessible, responsive services. Interventions such as hospital-based opportunistic immunisation [11], domiciliary immunisation [12], following up and reminding parents [13] about due immunisations are effective, and continue to be important in improving coverage. Here, governments must take responsibility to maximise the opportunities to vaccinate. However, such interventions do not address the (distinct) phenomenon of 'vaccine hesitancy' as we propose it should be defined.

The term 'vaccine hesitancy' should be applied to those parents whose conscious deliberations demonstrate something akin to indecision. However, the hesitancy concept will still not capture all who do not accept vaccines, since some parents may be decisive outright 'rejectors' of vaccines. Additionally, not all parents who have concerns refuse vaccines; in fact the vast majority of parents accept all vaccines on schedule despite some degree of concern. Hence we concur with the WHO working group figure, which puts hesitancy on a spectrum from full and partial to no vaccination [14].

Since vaccine uptake is affected by both acceptance and the logistical or opportunity-related factors we described earlier, coverage figures cannot be a good indicator of vaccine 'hesitancy'. Coverage (or lack thereof) gives no indication of any hesitancy on the pathway to full immunisation. Rather, purpose-built instruments are the only way to measure vaccine hesitancy [15, 16, 17]. Instruments developed (or under development) have focused on attitudes, safety, efficacy and behavioural constructs, such as trust, rather than access issues, which is entirely appropriate. Ongoing development and validation of these instruments across different settings will be important. Our engagement with attitudinal factors affecting uptake may also be enhanced by the use of existing terms that have been psychometrically validated, such as "decisional conflict," which refers to a state of uncertainty about a course of action. Decisional conflict is an internationally accepted construct with a validated scale where certain scores correlate with action. Importantly, the measure is sensitive to vaccination-related interventions [18].

There are other ways to identify the determinants of vaccination that can potentially navigate the access and acceptance dichotomy more effectively, especially when working towards 
interventions to increase uptake. After reviewing studies, Thomson et al [19] identified five fundamental causes of under vaccination: challenges to Access, Affordability, Awareness, Acceptance and Activation, a taxonomy referred to as the " $5 \mathrm{As}$ ". These domains capture most determinants of vaccine uptake. Future research could validate their comprehensiveness, assess their relative contributions to under vaccination and evaluate their utility in informing the development of evidence based solutions to low coverage.

There is a need to develop and evaluate solutions, tailored to the challenges of vaccine hesitancy, which are distinct from those needed for logistical problems. Finding and agreeing upon terms to clearly distinguish between vaccine hesitancy and the other determinants of uptake is an important start. We would suggest under-vaccinated as the over-arching term capturing both those who are unvaccinated and partially vaccinated for any reason. Reasons for under-vaccination could then be further expanded into a set of constructs with good content and construct validity. Each needs development of different interventions to improve uptake. Only an accurate terminology can give us a clear idea of where the problem lies, allow us to assess its scale, and then focus our attention accordingly. This way, our interventions have the very best chance of success in making vaccines available to those who want them, and helping those who are uncertain about their vaccination decision.

\section{References}

[1] Pegorie M, Shankar K, Welfare WS, Wilson RW, Khiroya C, Munslow G, Fiefield D, Bothra V, McCann R. Measles outbreak in Greater Manchester, England, October 2012 to September 2013: epidemiology and control. Euro Surveill. 2014 Dec 11;19(49):20982.

[2] Clemmons NS, Gastanaduy PA, Fiebelkorn AP, Redd SB, Wallace GS, Centers for Disease Control and Prevention (CDC). Measles-United States, January 4-April 2, 2015. MMWR Morb Mortal Wkly Rep. 2015 Apr 17;64(14):373-6.

[3] Ramsay ME. Measles: the legacy of low vaccine coverage. Archives of disease in childhood. 2013 Oct 1;98(10):752-4.

[4] Piccirilli G, Lazzarotto T, Chiereghin A, Serra L, Gabrielli L, Lanari M. Spotlight on measles in Italy: why outbreaks of a vaccine-preventable infection continue in the 21st century. Expert review of anti-infective therapy. $2015 \mathrm{Mar}$ 4;13(3):355-62.

[5] Dube, E., et al. Vaccine Hesitancy: An Overview. Human Vaccines and Immunotherapeutics, 2013. 9(8): p. 1763-1773.

[6] World Health Organization. Report of the SAGE working Group on vaccine hesitancy. 2014 
http://www.who.int/immunization/sage/meetings/2014/october/1_Report_WORKING_GR OUP_vaccine_hesitancy_final.pdf

[7] Samad L, Butler N, Peckham C, Bedford H, the Millennium Cohort Study Child Health Group. Incomplete immunisation uptake in infancy: maternal reasons. Vaccine 2006 Nov 17;24(47-48):6823-9.

[8] Pearce A, Marshall H, Bedford H, Lynch J. Barriers to childhood immunisation: Findings from the Longitudinal Study of Australian Children. Vaccine. 2015 Jun 26;33(29):3377-83.

[9] Nadeau JA, McNutt LA, Shaw J. Vaccination Coverage Rates and Factors Associated With Incomplete Vaccination or Exemption Among School-age Children Based in Public Schools in New York State. JAMA Pediatrics. 2016 Nov 1;170(11):1104-7.

[10] Roberts RJ, McGowan A, Cottrell S. Measuring inequalities in immunization in Wales and the impact of interventions. Human Vaccines \& Immunotherapeutics. 2016 Oct 2;12(10):2704-6.

[11] Shingler S, Hunter K, Romano A, Graham D. Opportunities taken: The need for and effectiveness of secondary care opportunistic immunisation. Journal of paediatrics and child health. 2012 Mar 1;48(3):242-6.

[12] Bond LM, Nolan TM, Lester RA. Home vaccination for children behind in their immunisation schedule: A randomised controlled trial. Med J Aust. 1998; 168:487-90; PMID:9631672

[13] Jacobson Vann JC, Szilagyi P. Patient reminder and recall systems to improve immunization rates. The Cochrane Library. 2005.

[14] MacDonald NE. Vaccine hesitancy: Definition, scope and determinants. Vaccine. 2015 Aug 14;33(34):4161-4.

[15] Opel DJ, Taylor JA, Mangione-Smith R, Solomon C, Zhao C, Catz S, Martin D. Validity and reliability of a survey to identify vaccine-hesitant parents. Vaccine. 2011 Sep 2;29(38):6598605.

[16] Larson HJ, Jarrett C, Schulz WS, Chaudhuri M, Zhou Y, Dube E, Schuster M, MacDonald NE, Wilson R. Measuring vaccine hesitancy: the development of a survey tool. Vaccine. 2015 Aug 14;33(34):4165-75.

[17] Gilkey MB, Magnus BE, Reiter PL, McRee AL, Dempsey AF, Brewer NT. The Vaccination Confidence Scale: a brief measure of parents' vaccination beliefs. Vaccine. 2014 Oct 29;32(47):6259-65.

[18] Shourie S, Jackson C, Cheater FM, Bekker HL, Edlin R, Tubeuf S, Harrison W, McAleese E, Schweiger M, Bleasby B, Hammond L. A cluster randomised controlled trial of a web based 
decision aid to support parents' decisions about their child's Measles Mumps and Rubella (MMR) vaccination. Vaccine. 2013 Dec 5;31(50):6003-10.

[19] Thomson A, Robinson K, Vallée-Tourangeau G. The 5As: A practical taxonomy for the determinants of vaccine uptake. Vaccine. 2016 Feb 17;34(8):1018-24. 